\title{
063
}

\section{EPIDEMIOLOGIA DELLE ESBL A LEGNANO DAL 2000 AL 2004 SU I5.094 GRAM NEGATIVI}

Vigano' EF., Bertinotti L., Agrappi C., Clerici P., Colombo A., De Paschale M., Mirri P., Barzani M.

U.O. Microbiologia, Ospedale Civile di Legnano, Via Candiani 2, 20025 Legnano (MI)

Introduzione Scopo del lavoro è la descrizione della diffusione di ESBL a Legnano, sia in enterobatteri che in altri Gram negativi.

Metodi Ricerca di ESBL con doppio disco su tutti i batteri 
Gram negativi e conteggio di un solo ESBL per paziente per ricovero e per specie batterica.

Risultati Su 15.094 batteri Gram negativi studiati sono state trovate 424 ESBL, pari al 2,81\%. Scomponendo l'osservazione in due periodi, è stato osservato che le ESBL erano il $2,33 \%$ da Maggio 2000 a Giugno 2002 ed erano il 4,48 \% nel 2004, con un incremento del $92 \%$. Considerando le sole Enterobacteriaceae la \%di ESBL dal 2002 al 2004 è raddoppiata ( dal 2,27 al 4,55\%).

La \% di ESBL all'interno delle specie è del $28 \%$ per P.stuar$t i$, del 11,54 \% per P.mirabilis e del 6,51\% per Enterobacter spp. Rilevante appare l'incremento dal 2000 al 2004 della \% di ESBL in E.coli, che è passata dallo $0,78 \%$ dei ceppi al $2,93 \%$, con un incremento di oltre 4 volte.

Una analisi in dettaglio nel 2004 ha evidenziato che il $50 \%$ dei casi di ESBL sono state ritrovate in ceppi isolati da pazienti esterni e in particolare da urine $(72 \%)$.

Nel 2004 i tassi di ESBL erano di 4,71 casi x 10.000 giornate di degenza: si tratta di tassi di poco inferiori a quelli osservati per MRSA (5,56 casi x 10.000 giornate).

Conclusioni L'Epidemiologia delle ESBL a Legnano è in linea con i dati nazionali: si conferma l'alta frequenza di casi in P.mirabilis e la tendenza all'aumento del numero assoluto di ESBL in tutte le Enterobacteriaceae, nelle quali è indispensabile la ricerca e conferma di ESBL con test fenotipici come doppio disco o altri. 